\title{
Oligoclonal banding of IgG in CSF, blood-brain barrier function, and MRI findings in patients with sarcoidosis, systemic lupus erythematosus, and Behçet's disease involving the nervous system
}

\author{
B N McLean, D Miller, E J Thompson
}

\begin{abstract}
$A$ retrospective study of CSF and serum analysis from a total of 43 patients with sarcoidosis, 20 with systemic lupus erythematosus, and 12 with Behçet's disease with neurological involvement found local synthesis of oligoclonal IgG using isoelectric focusing and immunoblotting in $51 \%, 25 \%$, and $8 \%$ respectively at some stage in their disease. Blood-brain barrier breakdown, when assessed with an albumin ratio found $47 \%$ of patients with sarcoidosis, $30 \%$ of those with systemic lupus erythematosus, and $42 \%$ of patients with Behçet's disease exhibiting abnormal barrier function at some time. Serial CSF analysis showed that clinical relapses were associated with worsening barrier function and in some patients the development of local oligoclonal IgG synthesis; conversely steroid treatment led to a statistically significant improvement in barrier function, and in two patients a loss of oligoclonal IgG bands.
\end{abstract}

A higher proportion of patients had MRI abnormalities than oligoclonal IgG or blood-brain barrier breakdown, MRI being abnormal in 16 of 19 patients with sarcoidosis, three of four patients with systemic lupus erythematosus, and seven of nine patients with Behçet's disease, although this may have been due to temporal factors.

In the differential diagnosis of chronic neurological disorders, locally synthesised oligoclonal IgG cannot distinguish between diseases, but the loss of bands seen in two patients contrasts with what is seen in multiple sclerosis, and thus may be a useful diagnostic clue.

Department of Neurochemistry B N McLean E J Thompson

University Department of Clinical Neurology, Institute of Neurology, Queen Square, London, UK D Miller

Correspondence to: Dr B N McLean, Department of Neurology, The Royal Cornwall Truro TR1 3LJ, UK. Received 29 April 1994 and in revised form 20 January 1995. Accepted 27 January 1995

$(尹$ Neurol Neurosurg Psychiatry 1995;58:548-554)

Keywords: IgG; sarcoidosis, Behçet's disease; systemic lupus erythematosis; magnetic resonance imaging

Ever since abnormalities of CSF, particularly with respect to immunoglobulin G (IgG), were first described by Kabat et al in the 1940 s in various neurological conditions, ${ }^{1}$ the range of neurological disorders associated with these abnormalities has been the subject of much study. ${ }^{2-5}$ The presence of what is thought to be locally synthesised IgG within the intrathecal compartment has been shown both quantitatively, and qualitatively with the description of oligoclonal banding of the IgG. The association of local synthesis of IgG, both quantitative and qualitative, with multiple sclerosis and infections of the nervous system has now been well established in many studies, but there is no such consensus on the inflammatory disorders, especially sarcoidosis and the connective tissue disorders when there is neurological involvement.

The neurological presentation of these disorders may be similar and can lead to great diagnostic difficulty in differentiating them from multiple sclerosis, especially when the neurological manifestations may be the first sign of the disease, as happens in about $5 \%$ of patients with sarcoidosis. ${ }^{6} 7$ Any laboratory investigation that can distinguish between the disorders would therefor be of great benefit, particularly in view of the potential for treatment of the inflammatory disorders. It has been reported that sarcoidosis is noted for the absence of local synthesis of oligoclonal IgG, and this has been suggested as a possible distinguishing feature. ${ }^{8}$ We have thus sought to define the incidence of CSF abnormalities in these inflammatory disorders by a retrospective analysis of all the definite cases referred to our laboratory for routine CSF analysis.

Magnetic resonance imaging has been shown to be of value in patients with inflammatory diseases of the CNS, particularly sarcoidosis. ${ }^{9}$ We have also sought to compare MRI data with the CSF data to examine the association between abnormalities found by the techniques, and to compare the sensitivities of the analyses.

\section{Materials and methods} PATIENTS

All cases of definite sarcoidosis, systemic lupus erythematosus, and Behçet's disease with neurological involvement referred for routine CSF analysis between 1986 and 1988 were included in the study. Sarcoidosis had been confirmed in all cases by biopsy, systemic lupus erythematosus by immunological parameters and clinical criteria, ${ }^{10}$ and Behçet's disease by clinical findings of oral and genital ulcers, ocular involvement, and appropriate neurological disturbance. Table 1 summarises the main neurological 
Table 1 Predominant neurological features in the study population. Where two or more features were equally prominent all were included

\begin{tabular}{|c|c|c|c|c|c|}
\hline $\begin{array}{l}\text { Sarcoidosis } \\
\text { Symptom/sign }\end{array}$ & No & $\begin{array}{l}\text { Systemic lupus } \\
\text { erythematosus } \\
\text { Symptom/sign }\end{array}$ & No & $\begin{array}{l}\text { Behçet's } \\
\text { Symptom/sign }\end{array}$ & No \\
\hline $\begin{array}{l}\text { Cortical sensory } \\
\text { or motor }\end{array}$ & 12 & $\begin{array}{l}\text { Dementia } \\
\text { Myelopathy }\end{array}$ & $\begin{array}{l}4 \\
4\end{array}$ & $\begin{array}{l}\text { Infarction } \\
\text { Meningitis }\end{array}$ & $\begin{array}{l}4 \\
3\end{array}$ \\
\hline Myelopathy & 10 & Encephalitis & 3 & Cerebellar & 3 \\
\hline Optic nerve & 8 & Cortical motor & 3 & Dementia & 2 \\
\hline Cerebellar & 7 & Optic nerve & 3 & Myelopathy & 1 \\
\hline Neuropathy & 5 & Cerebellar & 3 & Cranial nerve & 1 \\
\hline Cranial nerve & 3 & Cranial nerve & 3 & Optic nerve & 1 \\
\hline Cerebral mass & 3 & Meningitis & 1 & & \\
\hline Myopathy & 2 & Neuropathy & 1 & & \\
\hline Epilepsy & 2 & Epilepsy & i & & \\
\hline Hydrocephalus & 2 & & & & \\
\hline Meningitis & 2 & & & & \\
\hline Dementia & 1 & & & & \\
\hline Intracranial calcification & 1 & & & & \\
\hline Pituitary & 1 & & & & \\
\hline
\end{tabular}

abnormalities. Neurological involvement was confirmed by appropriate clinical manifestations, electrophysiology, imaging techniques, and biopsy of relevant tissue when feasible. Patients who did not have definite evidence that the neurological disturbance was associated with the systemic disease were excluded.

SPECIMENS

All CSF samples were obtained by lumbar puncture and paired CSF and serum samples were stored at $4^{\circ} \mathrm{C}$ and analysed within 24 hours of receipt.

\section{CELLULAR CONTENT OF CSF}

White and red cell counts were obtained directly by light microscopy. Samples contaminated by excess red blood cells $\left(>100 / \mathrm{mm}^{3}\right)$ were excluded from blood-brain-CSF barrier assessments. Differential white cell counts were not considered in this study.

INTACTNESS OF BLOOD-BRAIN BARRIER

Two methods were used to examine the intactness of the blood-brain barrier: $(a)$ total protein was measured by turbidimetry with benzethonium chloride precipitation. ${ }^{11}$ (b) Albumin concentrations were determined with a "monorocket" electroimmunoassay technique. ${ }^{12}$ An index of blood-brain barrier function was obtained by calculation of an albumin ratio, in which a CSF albumin $\times 10^{3}$ : serum albumin ratio of $>7$ is considered abnormal. ${ }^{13}$

\section{ASSESSMENT OF THE HUMORAL IMMUNE RESPONSE}

Three methods were used to examine the humoral immune response.

\section{IgG content}

Content of IgG was determined by a monorocket electroimmunoassay technique. ${ }^{12}$ This was used solely to determine the sample loading for isoelectric focusing and not for the calculation of indices, as we have previously shown that such indices are inferior to qualitative assessments, not only in multiple sclerosis but in other inflammatory and infectious disorders of the nervous system. ${ }^{1415}$
Isoelectric focusing of IgG

Isoelectric focusing for the detection of oligoclonal IgG was performed by the method of Walker et al. ${ }^{16}$ Briefly, $0.2 \mu \mathrm{g}$ IgG from CSF and diluted serum were loaded on to agarose gels, $\mathrm{pH}$ gradient $3-10 \cdot 5$, and isoelectric focusing was performed in a Pharmacia/LKB tank for $1500 \mathrm{~V} /$ hours at maximum $1500 \mathrm{~V}$, $20 \mathrm{~W}, 150 \mathrm{~mA}$. Protein was transferred by squashing on to nitrocellulose membranes, which were then incubated with goat antihuman Fc-specific IgG and rabbit antigoat IgG-horse radish peroxidase conjugate before visualising with 3-amino 4-ethyl carbazole. Serial samples, when available, were run on the same gel for comparison.

Local synthesis of IgG was defined in our laboratory as three or more bands present in the CSF by isoelectric focusing but not matched in serum. This definition we have now changed in the light of experience and with improved reagents, to two or more bands not arising from a single clone, but as the study was retrospective we have kept to our original criteria at the time of analysis. The relevance of single CSF bands has never been established, and because this does not meet the definition of oligoclonal, it was to be specifically excluded; in the event, no such single or even dual clones were found. An identical pattern seen in CSF and serum was considered to represent leakage of clones from the serum-a so called mirror pattern.

\section{Free light chain analysis}

The presence of free light chains was assessed by polyacrylamide gel electrophoresis of CSF, and kappa and lambda light chain immunoblotting was performed as previously described. ${ }^{17}$ Briefly, CSF specimens were electrophoresed on $120 \times 6 \mathrm{~mm}$ rod polyacrylamide gels for two hours with a uniform amount of protein (100 ng) loaded on each gel. Protein was transferred from the rod gels by compression on to nitrocellulose membranes, which were then incubated with type-specific goat antihuman antiserum and finally rabbit antigoat IgG-horse radish peroxidase conjugate before visualising with 3-amino 4-ethyl carbazole. Free light chains were assessed by counting the number of bands seen outside the heavy chain region on the gel immunoblots (and thus unbound); each band was assumed to represent a different B lymphocyte clone.

\section{MRI SCANS}

Patients were studied on a $0.5 \mathrm{~T}$ MRI scanner provided by the Multiple Sclerosis Society of Great Britain and Northern Ireland. Consecutive five or $10 \mathrm{~mm}$ axial slices of the whole brain were obtained with a moderately T2 weighted sequence (E2000/60). The size of brain lesions was quantified by a technique previously described. ${ }^{18}$ The lesions were divided into two subgroups according to their anatomical location: either contiguous with or separate from CSF containing spaces (subarachnoid spaces or the ventricular system). The sum of the lesion sizes was used 
to assess the total bulk of lesions in each location.

\section{Results \\ CSF ANALYSIS \\ Sarcoidosis}

A total of $69 \mathrm{CSF}$ and serum pairs from 43 patients were analysed. Tables 2 and 3 detail the isoelectric focusing and blood-brain barrier function results respectively for the individual CSF and serum pairs. All samples had isoelectric focusing performed, and 25 out of 69 (36\%) had local synthesis of oligoclonal IgG. One of the $69 \mathrm{CSF}$ samples was insufficient for albumin ratio analysis, which was abnormal in 38 of the remaining 68 (55\%) CSF and serum pairs. When there was local synthesis of oligoclonal IgG the bloodbrain barrier abnormality tended to be mild; absence of local synthesis was associated with more severe blood-brain barrier damage; however, there was no overall correlation between blood-brain barrier state and the presence of local synthesis of oligoclonal IgG.

Tables 2 and 3 also show the most abnormal responses that each individual patient recorded. In only one patient was there insufficient CSF for albumin analysis. Twenty of the $42(48 \%)$ patients had abnormal blood-brain barrier function at some time (representing $47 \%$ of the total number of patients), and 22 of the $43(51 \%)$ patients had local synthesis of oligoclonal IgG. In most patients with more than one CSF and serum pair analysis, the most abnormal blood-brain barrier function coincided with the most abnormal isoelectric focusing result. Ten

Table 2 Qualitative detection of intrathecal $\operatorname{IgG}$ production by isoelectric focusing and immunoblotting.

\begin{tabular}{llll}
\hline$I E F$ & Sarcoidosis & SLE & Behçet's \\
\hline A: & & & \\
Local synthesis & $25(36)$ & $7(28)$ & 2 \\
Serum leak & $3(4)$ & $7(28)$ & 1 \\
$\quad$ Negative & $41(59)$ & $11(44)$ & 11 \\
Total & 69 & 25 & 14 \\
B: & & & \\
Local synthesis & $22(51)$ & $5(25)$ & 1 \\
Serum leak & $2(5)$ & $7(35)$ & 1 \\
$\quad$ Negative & $19(44)$ & $8(40)$ & 10 \\
Total & 43 & 20 & 12 \\
\hline
\end{tabular}

Results are numbers of patients (\%). A = results from all CSF and serum pairs; $B=$ results from the most abnormal analysis for each patient. IEF = isoelectric focusing; SLE = systemic lupus erythematosus.

Table 3 Blood-brain barrier function assessed by the albumin ratio

\begin{tabular}{llcr}
\hline Blood-brain barrier function & Sarcoidosis & $S L E$ & Behçet's \\
\hline A: Normal & $30(43)$ & $17(68)$ & 8 \\
Abnormal & $38(55)$ & $6(24)$ & 5 \\
Insufficient & $1(1)$ & $2(8)$ & 1 \\
$\quad$ Total & 69 & 25 & 14 \\
B: & $22(51)$ & $12(60)$ & 7 \\
Normal & $20(47)$ & $6(30)$ & 5 \\
Abnormal & $1(2)$ & $2(10)$ & 0 \\
Insufficient & 43 & 20 & 12 \\
Total & & & \\
\hline
\end{tabular}

Results are numbers of patients (\%). $\mathrm{A}=$ Results from all CSF and serum pairs; $\mathrm{B}=$ results from the most abnormal analysis for each patient.

SLE $=$ Systemic lupus erythematosus. patients had serial CSF and serum analyses performed over periods of up to four years (data not shown). The development of neurological problems or clinical relapse were associated with worsening blood-brain barrier damage, an increase in the CSF total protein and white blood cell count and the development of local synthesis of oligoclonal IgG. Treatment with steroids or immunosuppressants was given to seven of the 10 patients, and in all resulted in improved blood-brain barrier function, reduction in CSF total protein and white blood cell count, and in one patient loss of local synthesis of oligoclonal bands. In two patients, however, treatment, with improvement in blood-brain barrier function, saw the development of local synthesis of oligoclonal bands.

Eight of the 25 patients with local synthesis of oligoclonal IgG produced a free light chain response, of which four were mixed kappa and lambda, three lambda alone, and one kappa alone. There seemed to be no relation between treatment or clinical relapse and the presence of a light chain response. One patient, negative for local synthesis of IgG, but with free light chains in the CSF, was found to have local synthesis of IgA with immunoblotting. This was the only patient examined for an IgA response.

In total $16 \mathrm{CSF}$ and serum pairs from nine patients had both normal isoelectric focusing and albumin ratios.

Systemic lupus erythematosus

A total of 25 CSF and serum pairs from 20 patients were analysed. Tables 2 and 3 show the individual paired results, with two pairs having insufficient CSF for the albumin ratio. Six of the $23(26 \%)$ samples had abnormal blood-brain barrier function (representing $24 \%$ of all sample pairs), and seven out of 25 (28\%) had local synthesis of oligoclonal IgG. None of six samples with local synthesis had abnormal blood-brain barrier function; six of 17 samples with no local synthesis had abnormal blood-brain barrier function. The relation between blood-brain barrier state and local synthesis of oligoclonal IgG was similar to sarcoidosis, with local synthesis associated with little or no impairment of the bloodbrain barrier and more severe impairment with the absence of local synthesis.

Tables 2 and 3 show the most abnormal responses that each patient recorded. Two patients did not have enough CSF for an albumin ratio calculation. Six out of 18 patients had abnormal blood-brain barrier function at some time, and five out of $20 \mathrm{had}$ local synthesis of oligoclonal IgG.

Four patients had serial CSF and serum analyses performed over periods up to 14 months (data not shown). Clinical relapse was associated with worsening impairment of the blood-brain barrier, an increase in CSF total protein, and in one patient the development of serum oligoclonal IgG. Treatment was associated in another patient with an improvement in blood-brain barrier function and a reduction in free light chain synthesis, and in 
yet another patient with the loss of local synthesis of oligoclonal IgG. Two patients remained negative for locally synthesised oligoclonal IgG, although one developed a mirror pattern during relapse.

Only three patients had a free light chain response. With such small numbers, no overall comment can be made as to any relation with disease activity. Four patients with systemic lupus erythematosus had a normal CSF and serum analysis by isoelectric focusing and albumin ratio.

Behçet's disease

Fourteen CSF and serum pairs from 12 patients with Behçet's disease were analysed (tables 2 and 3). Five out of 13 pairs, or five out of 12 patients, had abnormal blood-brain barrier function. Only two out of 14 pairs, or one out of 12 patients had local synthesis of IgG.

Two patients had two CSF and serum pairs analysed (data not shown). In one patient, steroid treatment resulted in an improvement of blood-brain barrier function and a reduction in the white blood cell count, but no alteration in the isoelectric focusing pattern. In the other the initial CSF was normal, but only oligoclonal band and free light chain analyses were performed on the second CSF, which remained normal despite disease progression requiring treatment.

No patient produced a free light chain response in the CSF, and six patients had a normal albumin ratio and isoelectric focusing analysis.

CSF CHANGES IN RESPONSE TO TREATMENT

Combining the results for the effect of steroids and immunosuppressants on the CSF variables studied in all three groups of patients, there was a statistically significant association between the introduction of such drugs and an improvement in blood-brain barrier function when assessed by the albumin ratio (Fisher's exact test; $P=0.05$ ) a reduction in CSF total protein (Fisher's exact test; $P=0.018$ ), and an improvement in CSF white blood cell count (Fisher's exact test, $P=0.018$ ). There was no significant association with changes on isoelectric focusing.

MAGNETIC RESONANCE IMAGING

Table 4 gives a summary of the results of MRI scanning with respect to the presence or absence of abnormalities and the correlation

Table 4 Comparison of MRI results in all three groups of diseases with isoelectric focusing result and blood-brain barrier function assessed by the albumin ratio.

\begin{tabular}{|c|c|c|c|c|c|c|}
\hline & \multicolumn{2}{|c|}{ Sarcoidosis } & \multicolumn{2}{|l|}{$S L E$} & \multicolumn{2}{|c|}{ Behçet's } \\
\hline & $+v e$ & $-v e$ & $+v e$ & $-v e$ & $+v e$ & $-v e$ \\
\hline \multicolumn{7}{|c|}{ Isoelectric focusing } \\
\hline MRI + ve & 11 & 5 & 2 & 1 & 1 & 6 \\
\hline MRI - ve & 1 & 2 & 0 & 1 & 0 & 2 \\
\hline \multicolumn{7}{|c|}{ Blood-brain barrier } \\
\hline MRI + ve & 7 & 9 & 3 & 0 & 5 & 2 \\
\hline MRI - ve & 1 & 2 & 0 & 1 & 0 & 2 \\
\hline
\end{tabular}

+ ve = abnormal; $-\mathrm{ve}=$ normal; SLE $=$ systemic lupus erythematosus. with isoelectric focusing and blood-brain barrier function when assessed by polyacrylamide gel electrophoresis.

\section{Sarcoidosis}

Nineteen patients underwent brain MRI examinations, of which 16 were abnormal. Only 12 of the 19 had local synthesis of IgG, and eight had abnormal blood-brain barrier function. Of the 16 patients with abnormal MRIs, 11 had local synthesis of IgG, and seven abnormal blood-brain barrier function.

Sixteen patients had quantitative MRI analysis performed. Thirteen were abnormal, 12 of the 16 had lesions "contiguous with CSF", 10 had lesions "separate from CSF", nine had both "contiguous" and "separate" lesions, three "contiguous" lesions only, and one "separate" lesions only.

There was no statistical association between the site of the lesions on MRI and the presence of local synthesis of IgG or blood-brain barrier function, or between total volume of abnormality on MRI and degree of blood-brain barrier impairment assessed by the albumin ratio. The exclusion of patients with MRI and CSF analysis more than six weeks apart or with MRI performed later than the CSF analysis improved the statistical associations but these did not quite achieve significance. It should be noted that 10 patients had a myelopathy as part of their disease, and in five this was the only neurological disturbance. Five patients with myelopathy had brain MRI, three with myelopathy alone, and all were positive on MRI. Two of the three patients with negative MRI had optic neuropathies.

\section{Systemic lupus erythematosus}

Only four patients underwent MRI, of which three were abnormal. Two of these three had local synthesis of IgG, and all had abnormal blood-brain barrier function.

There was no statistical association between MRI findings and local synthesis of IgG or blood-brain barrier function.

\section{Behçet's disease}

Nine patients underwent MRI examinations, and all had quantitative analyses.

Seven of the nine patients had abnormal MRI. Only one of the nine had local synthesis of IgG, and five had abnormal blood-brain barrier function. Of the seven abnormal on MRI, only one had local synthesis of IgG, and five had abnormal blood-brain barrier function.

Six patients had "separate" lesions, three had "contiguous" abnormalities, two had abnormalities of both contiguous and separate nature, one contiguous only and four separate only.

There was no statistical association between site or volume of lesions and local synthesis of IgG or blood-brain barrier functions.

Effect of interval between lumbar puncture and $M R I$ on result concordance

A comparison of the interval between lumbar puncture and MRI for each diagnostic group, 
and agreement (both abnormal or normal) or disagreement (one normal, the other abnormal) in the results was performed. Overall, in all three disease groups, there was no statistical association between result agreement or disagreement and the MRI examination taking place before or after the lumbar puncture. When sarcoidosis alone was considered, there was a significant association between MRI being performed after the lumbar puncture, and disagreement in the results of isoelectric focusing (Fishers exact test, $P=0.049$ ), but not blood-brain barrier function. In the other two disease groups taken individually, there were no statistical associations found, but numbers were small.

\section{Discussion}

A review of the medical literature to date concerning the presence of intrathecally synthesised IgG in the three disorders with neurological involvement has yielded information on only 10 patients with Behçet's disease, 42 patients with systemic lupus erythematosus, and 53 patients with sarcoidosis (table 5). ${ }^{19-36}$ Most studies have used agarose electrophoresis for the detection of oligoclonal IgG, with only a few using polyacrylamide gel isoelectric focusing, one study using PAGE, and only one other study using agarose isoelectric focusing as we have. A variety of quantitative methods were also employed, predominantly the IgG index, although the reference ranges for the upper limit of normal varied from 0.58 to 0.83 . Many patients

Table 5 Summary of previous reports on intrathecal IgG synthesis in sarcoidosis, systemic lupus erythematosus, and Behçet's disease involving the CNS

\begin{tabular}{|c|c|c|c|c|c|}
\hline $\begin{array}{l}\text { Patient } \\
\text { Nos }\end{array}$ & $\begin{array}{l}I E F \\
+v e\end{array}$ & $\begin{array}{l}\text { Age } \\
+v e\end{array}$ & Other +ve & Reference & Year \\
\hline \multicolumn{6}{|c|}{ Sarcoidosis: } \\
\hline 2 & & 1 & \multirow{4}{*}{$1 \mathrm{IgG} / \mathrm{alb}$} & Miller et al ${ }^{19}$ & 1983 \\
\hline 1 & & 1 & & Bloomer and Bray ${ }^{20}$ & 1981 \\
\hline 1 & & 0 & & Johnson et al ${ }^{21}$ & 1980 \\
\hline 2 & & 1 & & Ebers and Paty ${ }^{22}$ & 1980 \\
\hline 2 & & & \multirow{2}{*}{$\begin{array}{l}1 \text { IgG-ind } \\
2 \text { IgG-syn } \\
4 \text { IgG-ind } \\
4 \text { IgG-syn }\end{array}$} & Caroscio et $a l^{23}$ & 1983 \\
\hline 5 & & 0 & & Borucki et al ${ }^{8}$ & 1989 \\
\hline 25 & & & \multirow{3}{*}{$\begin{array}{l}7 \mathrm{IgG}-\text {-ind } \\
0 \mathrm{IgG} \text {-ind } \\
1 \mathrm{IgG}-\text { ind } \\
2 \mathrm{IgG}-\mathrm{syn}\end{array}$} & Oksanen $^{24}$ & 1986 \\
\hline 7 & & $0 / 7$ & & Stern et $a l^{25}$ & 1987 \\
\hline 5 & & $0 / 3$ & & Mitchell et $a l^{26}$ & 1984 \\
\hline 1 & \multirow[t]{3}{*}{$1(\mathrm{P})$} & & 1 IgG-ind & Kinnman and Link ${ }^{27}$ & 1984 \\
\hline 1 & & $1(P)$ & 0 IgG-ind & Perkin $e t a l^{28}$ & 1983 \\
\hline$?$ & & & $1 \mathrm{Ig}$-prot & Yahr et $a l^{2}$ & 1954 \\
\hline \multicolumn{6}{|l|}{ SLE: } \\
\hline 1 & & 1 & \multirow{6}{*}{$\begin{array}{l}0 \text { IgG-ind } \\
3 \mathrm{IgG}-\text { syn } \\
4 \mathrm{IgG} / \mathrm{alb} \\
0 \mathrm{IgG} \text {-ind } \\
5 \mathrm{IgG} \text {-ind } \\
5 \text { Alb-rat }\end{array}$} & Miller et al ${ }^{19}$ & 1983 \\
\hline 1 & $1(P)$ & 1 & & Hosein and Johnson ${ }^{29}$ & 1981 \\
\hline 4 & 3 (A) & & & Link and Kostulas ${ }^{30}$ & 1983 \\
\hline 11 & & 2 & & Bloomer and Bray ${ }^{20}$ & 1981 \\
\hline 1 & & 0 & & Olsson and Pettersson ${ }^{31}$ & 1976 \\
\hline 19 & $8(P)$ & & & Winfield et al ${ }^{32}$ & 1983 \\
\hline 2 & \multirow{3}{*}{$0(\mathrm{P})$} & 0 & \multirow{3}{*}{1 IgG-ind } & Ebers ${ }^{22}$ & 1980 \\
\hline 2 & & 1 & & Hershey and Trotter ${ }^{33}$ & 1980 \\
\hline 1 & & 0 & & Rudick et al $^{34}$ & 1985 \\
\hline \multicolumn{6}{|c|}{ Behçet's: } \\
\hline 2 & & 1 & \multirow{5}{*}{$1 \mathrm{Ig}$-prot } & Miller et al ${ }^{19}$ & 1983 \\
\hline 2 & $0(\mathrm{P})$ & 1 & & Hosein and Johnson ${ }^{29}$ & 1981 \\
\hline$?$ & & & & Yahr et $a l^{2}$ & 1954 \\
\hline 1 & & 1 & & Schoenen and Dalwaide ${ }^{35}$ & 1975 \\
\hline 4 & & 2 & & Gille et $a^{36}$ & 1990 \\
\hline
\end{tabular}

(P) = polyacrylamide; $(\mathrm{A})=$ agarose; IgG-ind = IgG-index; IgG-syn = IgG synthesis rate; $\mathrm{IgG} / \mathrm{alb}=\mathrm{IgG} /$ albumin ratio in $\mathrm{CSF} ; \mathrm{Alb}$-rat $=\mathrm{CSF}$ :serum albumin ratio; Ig-prot = gammaglobulin:total protein ratio. underwent examination by several methods, but the study by Yahr et $a l^{2}$ did not specify the total numbers studied.

Overall, local synthesis of oligoclonal IgG was found by any method in five out of nine patients with Behçet's disease, 16 out of 42 (38\%) patients with systemic lupus erythematosus, and five out of $23(22 \%)$ patients with sarcoidosis.

With quantitative methods, overall the one patient (out of an uncertain number) studied with Behçet's disease, 10 out of 37 (27\%) patients with systemic lupus erythematosus, and 18 out of $48(38 \%)$ patients with sarcoidosis (including the one patient reported by Yahr et $a l^{2}$ ) had intrathecal synthesis of IgG.

In our study, all patients underwent agarose isoelectric focusing with specific immunofixation for IgG, and most had calculation of an albumin ratio for determination of blood-brain barrier function.

We have found that $51 \%$ of patients with sarcoidosis have local synthesis of oligoclonal IgG, a figure more than double the proportion found in reported series, and $25 \%$ of patients with systemic lupus erythematosus have local synthesis of oligoclonal IgG, which is lower than that reported. Only $8 \%$ of patients with Behçet's disease in our study had local synthesis of oligoclonal IgG, but our 12 cases must be considered more representative than the total of 10 from the medical literature. The differences with the reported series may be due to the uniformity of the methods of analysis applied to our patients, and the small numbers of patients reported by most authors. Only two series have comparable numbers: Oskanen et al with 25 patients with sarcoidosis, ${ }^{24}$ and Winfield et al with 19 patients with systemic lupus erythematosus. ${ }^{32}$ Only in the second series was isoelectric focusing performed, finding $42 \%$ patients to have local synthesis of oligoclonal IgG.

Only two patients had serum bands in addition to locally synthesised oligoclonal IgGboth had sarcoidosis. A larger number had a mirror pattern, indicating passive transfer from serum - three sarcoidosis, eight systemic lupus erythematosus, and one Behçet's disease. This serum response is not unexpected in conditions that have a systemic basis and does not differentiate from multiple sclerosis where such responses occur in a small proportion of patients. A few patients were noted to have some bands with differential staining intensity between serum and CSF; sometimes the serum bands were stronger and sometimes the CSF bands, even in the same sample. An adequate explanation for this has not yet emerged, although selective recruitment of lymphocyte populations has been proposed.

Light chain analysis has not featured in literature reviews. We have found only a few patients (12 out of 75) producing excess free light chains, without any relation to disease activity and, with only one exception, no change with treatment. This contrasts with multiple sclerosis where raised free light chain 
numbers are reported to be associated with recent antigenic stimulation. ${ }^{37}$

One relevant issue is the influence of treatment on the CSF. In two patients the use of corticosteroids or immunosuppressants resulted in a loss of the oligoclonal response. The report from Gille et $a l^{36}$ also showed the loss of oligoclonal bands in a patient with Behçet's disease treated with steroids but information on treatment is not available from most of the other studies, so interpretation of the results have to be viewed with this in mind.

A large minority of patients with sarcoid, systemic lupus erythematosus, and Behçet's disease manifesting neurological problems had abnormal blood-brain barrier function, indicating appreciable disease activity involving the CNS, even when there was no evidence of intrathecal immunological stimulation as indicated by oligoclonal IgG synthesis. Most of these patients had mild to moderate blood-brain barrier impairment, but some severe responses were found in all disease groups. Although there was no apparent correlation between blood-brain barrier function overall and severity of clinical disease, serial samples in individual patients clearly displayed a temporal relation to clinical activity.

Steroids or immunosuppressants considerably reduced the permeability of the bloodbrain barrier, reflecting a "tightening up" of the blood-brain barrier, and clinical relapses saw an increased impairment in blood-brain barrier function. In some patients the improvement in blood-brain barrier function with treatment saw the appearance of local synthesis of oligoclonal IgG. Whether this represents an unmasking of the CSF bands by the removal of serum polyclonal IgG is difficult to prove conclusively, but it is the most likely explanation, and may again explain some of the published differences. The improvement in blood-brain barrier function must contribute to the clinical effects of immunomodulating agents irrespective of any effect that they may have on the primary disease process as evidenced in this study by the loss of oligoclonal IgG and changes in free light chains and white blood cell counts in some patients. This changing response of oligoclonal IgG may be useful diagnostically, in that patients with multiple sclerosis never lose their oligoclonal response, which remains constant over long periods. As the differential diagnosis often lies between multiple sclerosis and these disorders, such a change would suggest that multiple sclerosis is less likely, but further studies are required with larger patient numbers to assess how reliable this is.

Magnetic resonance imaging consistently yielded a greater proportion of patients with abnormalities in all three groups in this study. Great caution must be exercised, however, when comparing analyses performed at differing times in these diseases where neurological damage is a dynamic and intermittent process. As we have shown, in our patients with sarcoidosis MRI was more likely to produce a result differing from isoelectric focusing of IgG when it was performed later than the lumber puncture. This indicates that the reason for the better performance of MRI in this group may be due in part to temporal factors in the study, rather than to a greatly increased sensitivity. Two other factors may account for the discrepant MRI and CSF findings. Firstly, old MRI was nonenhanced-it is not possible on such a scan to distinguish old, inactive gliotic regions from active inflammatory or granulomatous regions. One could anticipate the second making the more important contribution to CSF abnormalities. Future studies should include gadolinium-DPTA enhanced MRI, as this agent can cross an abnormal blood-brain barrier and thus identify active inflammatory/ granulomatous lesions. Secondly, MRI of the spinal cord was not performed-it is possible that disease activity at this level contributes to the CSF abnormalities in some cases. Such considerations must therefore be borne in mind when examining retrospective studies of comparisons between MRI and CSF analysis in other diseases, notably multiple sclerosis. Furthermore, the appearances on unenhanced MRI of multiple sclerosis, systemic lupus erythematosus, sarcoidosis and Behçet's disease may be indistinguishable; ${ }^{98}$ in such a setting, the serial examination of CSF may help to clarify the diagnosis. Magnetic resonance imaging and CSF data may thus be regarded as complementary. There are other CSF variables that may be important in inflammatory disorders that were not examined here, such as cytokines and mononuclear cell populations, and comparisons of these with MRI may yield more fruitful results, but they were not within the scope of this study given its retrospective nature.

Our study reaffirms that local synthesis of oligoclonal IgG is a feature of sarcoidosis and systemic lupus erythematosus, and is uncommon in Behçet's disease when there is neurological involvement, and as such cannot be used to distinguish them from multiple sclerosis or other demyelinating disorders. The presence of a changing oligoclonal IgG response with clinical state and with treatment in a few patients needs further study. The nature of the antigen(s) exciting local synthesis of oligoclonal IgG is unknown, but the changing response to treatment implies that the antigen(s) are transitory, and may perhaps be "self" antigens revealed by destructive processes, as has been suggested for multiple sclerosis. Blood-brain barrier function is also impaired during active disease in most patients, and this impairment, which parallels clinical severity, can be modulated by immunosuppressive agents.

1 Kabat EA, Moore DH, Landow H. An electrophoretic study of the protein components in cerebrospinal fluid 1942;21:571-7.

2 Yahr MD, Goldensohn SS, Kabat EA. Further studies on the gamma globulin content of cerebrospinal fluid in multiple sclerosis and other neurological diseases. Ann NY Acad Sci 1954;58:613-24. 
3 Kostulas VK. Oligoclonal IgG bands in cerebrospinal fluid. Methodological and clinical aspects. Acta Neurol Scand 1985;72:suppl.

4 Link H, Muller R. Immunoglobulins in multiple sclerosis and infections of the nervous system. Arch Neurol $1971 ; 25: 326-44$

5 Vandvik B, Skrede S. Electrophoretic examination of cerebrospinal fluid proteins in multiple sclerosis and other neurological diseases. Eur Neurol 1973;9:224-41.

6 Stern BJ, Krumholz A, Johns C, Scott P, Nissim J. Sarcoidosis and its neurological manifestations. Arch Neurol 1985;42:909-17.

7 Delaney P. Neurological manifestations in sarcoidosis: review of the literature, with a report of 23 cases. Ann review of the literature, with
Intern Med 1977;87:336-45.

8 Borucki SJ, Nguyen BV, Ladoulis CT, McKendall RR. Cerebrospinal fluid immunoglobulin abnormalities in neurosarcoidosis. Arch Neurol 1989;46:270-3.

9 Miller DH, Kendall BE, Barter S, et al. Magnetic resonance imaging in central nervous system sarcoidosis. Neurology 1988;38:378-83.

10 Tan EM, Cohen AS, Fries JF, et al. The 1982 revised criteria for the classification of systemic lupus erythematosus. Arthritis Rheum 1982;35:1271-7.

11 Luxton R, Patel P, Keir G, Thompson EJ. A micromethod for measuring total protein in cerebrospinal fluid using benzethonium chloride in microtitre plate wells. Clin Chem 1989;35:1731-4.

12 Laurell C-B. Electroimmunoassay. Scand 7 Clin Lab Invest 1972;29:21-37.

13 Tibbling G, Link H, Ohman S. Principles of albumin and IgG analysis in neurological disorders, part 1 (establishment of reference values). Scand $\mathcal{f}$ Clin Lab Invest 1977;37:385-90.

14 McLean BN, Luxton RW, Thompson EJ. A study of immunoglobulin $G$ in the cerebrospinal fluid of 1007 patients with suspected neurological disease usin isoelectric focusing and the log IgG-index. Brain 1990; 113:1269-89.

15 Luxton RW, McLean BN, Thompson EJ. Isoelectric focusing versus quantitative measurements in the detection of intrathecal local synthesis of IgG. Clin Chim Acta 1990;187:297-308.

16 Walker RWH, Keir G, Johnson MH, Thompson EJ. A rapid method for detecting oligoclonal IgG in unconcentrated CSF, by agarose isoelectric focusing, transfer to trated CSF, by agarose isoelectric focusing, transfer to cellulose nitrate and imm

17 Thompson EJ, Keir G. Improved detection of oligoclonal and Bence-Jones proteins by $\mathrm{k} / \mathrm{l}$ immunoblotting. Clin Chim Acta 1984;143:329-35.

18 Ormerod IEC, Miller DH, McDonald WI, et al. The role of NMR imaging in the assessment of multiple sclerosis in isolated neurological lesions: a quantitative study. Brain 1987;110:1579-1616.

19 Miller JR, Burke AM, Bever CT. Occurrence of oligoclonal bands in multiple sclerosis and other CNS diseases. Ann Neurol 1983;13:53-8.

20 Bloomer LC, Bray PF. Relative value of three laboratory methods in the diagnosis of multiple sclerosis. Clin Chem 1981;27:2011-3.
21 Johnson KP, Likosky WH, Nelson BJ, Fein G. Comprehensive viral immunology of multiple sclerosis. Clinical, epidemiological, and CSF studies. Arch Neurol 1980;37:537-41.

22 Ebers GC, Paty DW. CSF electrophoresis in one thousand patients. F Can Sci Neurol 1980;7:275-80.

23 Caroscio JT, Kochwa S, Sacks H, Cohen JA, Yahr MD. Quantitative CSF IgG measurements in multiple sclerosis and other neurologic diseases. Arch Neurol 1983;40: 409-13.

24 Oksanen V. Neurosarcoidosis: clinical presentations and course in 50 patients. Acta Neurol Scand 1986;73: 283-90.

25 Stern BJ, Griffin DE, Luke RA, Krumholz A, Johns CJ. Neurosarcoidosis: cerebrospinal fluid lymphocyte subpopulations. Neurology 1987;37:878-81.

26 Mitchell JD, Yap PL, Milne LA, Lachmann PJ, Pentland B. Immunological studies on the cerebrospinal fluid in neurological sarcoidosis. $\mathcal{f}$ Neuroimmunology 1985;7 249-53.

27 Kinnman J, Link $H$. Intrathecal production of oligoclona IgM and IgG in CNS sarcoidosis. Acta Neurol Scand 1984;69:97-106.

28 Perkin GD, Sethi K, Muller BR. IgG ratios and oligoclonal IgG in multiple sclerosis and other neurological disorders. F Neurol Sci 1983;60:325-36.

29 Hosein ZZ, Johnson KP. Isoelectric focusing of cerebrospinal fluid proteins in the diagnosis of multiple sclerosis. Neurology 1981;31:70-6.

$30 \mathrm{Link} \mathrm{H}$, Kostulas V. Utility of isoelectric focusing of cerebrospinal fluid and serum on agarose evaluated for neurological patients. Clin Chem 1983;29:810-5.

31 Olsson J-E, Pettersson B. A comparison between agar ge electrophoresis and CSF serum quotients of IgG and albumin in neurological diseases. Acta Neurol Scand 1976;53:308-22.

32 Winfield JB, Shaw M, Silverman LM, et al. Intratheca IgG synthesis and blood-brain barrier impairment in patients with systemic lupus erythematosus and central nervous system dysfunction. Am $\mathcal{F} M e d 1983 ; 74$ : 837-44.

33 Hershey LA, Trotter JL. The use and abuse of the cerebrospinal fluid profile in the adult: a practical evaluation. Ann Neurol 1980;8:426-34.

34 Rudick RA, Peter DR, Biblack JM, Knutson DW Multiple sclerosis: free light chains in cerebrospinal Multiple sclerosis: free light chai
fluid. Neurology 1985;35:1443-9.

35 Schoenen J, Delwaide PJ. A propos d'une uveo-nevraxite. Acta Neurol Belg 1975;75:267-78.

36 Gille M, Sindic CJM, Laterre PF, et al. Atteintes neurologiques revelatrices d'une maladie de Behçet: quatres observations cliniques. Acta Neurol Belg 1990 90:233-47

37 Vakaet A, Thompson EJ. Free light chains in the cerebrospinal fluid: an indicator of recent immunological stimulation. $\mathcal{f}$ Neurol Neurosurg Psychiatry 1985;48. 995-8.

38 Miller DH, Ormerod IEC, Gibson A, et al. MR brain scanning in patients with vasculitis: differentiation from multiple sclerosis. Neuroradiology 1987;29: 226-31. 